\title{
BabyNoggin Pre-implementation Phase: Understanding How Clinical Teams and Parents Will Respond to BabyNoggin
}

\author{
Sarah W. Watkins ${ }^{1}$, Elizabeth Z. Chen ${ }^{2}$, Katie Swec ${ }^{1}$, Jordan Huskins ${ }^{1}$, Jin Lee ${ }^{3}$, \\ Andrew D. Miller ${ }^{4}$, Nerissa S. Bauer ${ }^{1}$ \\ ${ }^{1}$ Indiana University School of Medicine, ${ }^{2}$ University of Notre Dame, \\ ${ }^{3}$ BabyNoggin ${ }^{\mathrm{TM}},{ }^{4}$ Indiana University School of Informatics and Computing
}

Background and Purpose: Mobile health (mHealth) apps hold potential to transform healthcare, but their adoption in clinical settings takes time and how providers and families use them is unknown. Three clinics at Riley Hospital for Children are preparing to implement BabyNoggin, an app that collects developmental screening tools typically collected via paper forms. The purpose of this study was two-fold: 1) to understand pre-implementation processes and provider perspectives of integrating BabyNoggin into clinic workflow; and 2) to examine parental attitudes towards the use of apps for child development.

Project Methods: Pre-implementation clinical observations of workflow were performed in participating clinics. Semi-structured interviews informed by the Consolidated Framework for Implementation Research (CFIR) were conducted with each clinical team to understand perceived implementation facilitators and barriers. Lastly, parents with children aged 0-5 were recruited from study clinics and social media to participate in a 26-item survey to gauge their attitudes towards using apps in place of paper screening forms and for tracking their child's development.

Results: Implementation workflows were co-created with each clinical team that should not increase visit length. Five CFIR interviews highlighted facilitators and barriers towards implementation of BabyNoggin to be considered during implementation. Out of 199 parents who completed the survey, $72.9 \%(n=145)$ reported a higher likelihood of downloading a child health app that was recommended by their pediatrician.

Conclusion and Future Steps: This study constituted the pre-implementation phase of a larger project involving the implementation of BabyNoggin. Future research will use these findings as a guide during implementation. 